\title{
Statistical methods for the analysis of rotation measure grids in large scale structures in the SKA era
}

\author{
Vacca V. ${ }^{* a}$, Oppermann N. ${ }^{b}$, Enßlin T. ${ }^{a, c}$, Selig M. ${ }^{a, c}$, Junklewitz H. ${ }^{d}$, Greiner M. ${ }^{a, c}$, \\ Jasche J. ${ }^{e}$, Hales, C. A. ${ }^{f, g}$, Reinecke M. ${ }^{a}$, Carretti E. ${ }^{h}$, Feretti L. ${ }^{i}$, Ferrari C. ${ }^{j}$, \\ Giovannini G. ${ }^{i, k}$, Govoni F. ${ }^{l}$, Horellou C. ${ }^{m}$, Ideguchi S. ${ }^{n}$, Johnston-Hollitt M. ${ }^{o}$, Murgia \\ M. $^{l}$, Paladino R. ${ }^{i, k}$, Pizzo R. F. ${ }^{p}$, and Scaife A. ${ }^{q}$ \\ a Max Planck Institute for Astrophysics, Karl-Schwarzschild-Str. 1, 85748 Garching, Germany; \\ ${ }^{b}$ Canadian Institute for Theoretical Astrophysics, University of Toronto, 60 St. George Street, \\ Toronto ON, M5S 3H8, Canada; ${ }^{c}$ Ludwig-Maximilians - Universität München, \\ Geschwister-Scholl-Platz 1, 80539, MÃijnchen, Germany; ${ }^{d}$ Argelander-Institut für Astronomie, \\ Auf dem Hügel 71, 52121 Bonn, Germany; ${ }^{e}$ CNRS, UMR 7095, Institut d'Astrophysique de \\ Paris, 98 bis, boulevard Arago, F-75014 Paris, France; ${ }^{f}$ Jansky Fellow of the National Radio \\ Astronomy Observatory; ${ }^{g}$ National Radio Astronomy Observatory, PO Box 0, Socorro, NM \\ 87801, USA; ${ }^{h}$ CSIRO Astronomy and Space Science PO Box 276. Parkes 2870; ${ }^{i}$ INAF-Istituto \\ di Radioastronomia, Via P.Gobetti 101, 40129 Bologna, Italy; ${ }^{j}$ Université de Nice Sophia \\ Antipolis, CNRS, Observatoire de la Côte d'Azur, Laboratoire Cassiopée, Nice, France; ${ }^{k}$ \\ Department of Physics and Astronomy, University of Bologna, V.le Berti Pichat 6/2, 40127 \\ Bologna, Italy; ' INAF-Osservatorio Astronomico di Cagliari, Strada 54, Loc. Poggio dei Pini, \\ 09012 Capoterra (Ca), Italy; ${ }^{m}$ Department of Earth and Space Sciences, Chalmers University \\ of Technology, Onsala Space Observatory, SE-439 92 Onsala, Sweden; ${ }^{n}$ University of \\ Kumamoto, 2-39-1, Kurokami, Kumamoto 860-8555, Japan; ${ }^{\circ}$ School of Chemical \& Physical \\ Sciences, Victoria University of Wellington, PO Box 600, Wellington 6014, New Zealand; $p$ \\ ASTRON, Postbus 2, 7990 AA, Dwingeloo, The Netherlands; ${ }^{q}$ University of Southampton \\ Highfield, Southampton SO17 1BJ, U.K. \\ E-mail: vvacca@mpa-garching.mpg.de
}

To better understand the origin and properties of cosmological magnetic fields, a detailed knowledge of magnetic fields in the large-scale structure of the Universe (galaxy clusters, filaments) is crucial. We propose a new statistical approach to study magnetic fields on large scales with the rotation measure grid data that will be obtained with the new generation of radio interferometers.

Advancing Astrophysics with the Square Kilometre Array

June 8-13, 2014

Giardini Naxos, Italy

\footnotetext{
* Speaker.
} 


\section{Introduction}

Magnetic fields have been detected in planets, stars, galaxies, and clusters of galaxies. Intracluster magnetic fields are characterized by a strength of $\sim \mu \mathrm{G}$ and fluctuations on scales from a few kpc to $\sim 100 \mathrm{kpc}$ (e.g., Enßlin \& Vogt 2003, Vogt \& Enßlin 2003, Vogt \& Enßlin 2005, Murgia et al. 2004, Govoni et al. 2006, Guidetti et al. 2008, Bonafede et al. 2009, Bonafede et al. 2010, Vacca et al. 2010, Vacca et al. 2012). On larger scales, along galaxy filaments as well as in voids and sheets, a firm detection has not yet been possible. Cosmological magneto-hydro-dynamical simulations indicate that magnetic fields are present in intergalactic low-density environments (e.g., Dolag et al. 2008), while $\gamma$-ray observations suggest their presence in voids where a lower limit of $\sim 1 \mathrm{fG}$ has been derived (see Beck A. et al. 2013 and references therein). For more details of our current observational understanding of magnetic fields in low-density regions, see also the chapter by Giovannini et al. (2014) in these proceedings.

Magnetic fields can be studied using a variety of techniques, among which rotation measure (RM) observations are a powerful tool (e.g., Carilli \& Taylor 2002, Govoni et al. 2004). The amount of Faraday rotation measured from radio observations along a given line of sight is the result of the contribution from the Milky Way, the radio source itself and other extragalactic environments. The distribution of matter in large-scale structures can be classified into four types (following Hahn et al. 2007, using a criterion of local stability of test particle orbits): voids, sheets, filaments and galaxy clusters. We will hereafter call these four types, as well as the sources responsible for the radio emission, environments.

Disentangling the contributions from different extragalactic environments is essential in order to study magnetic fields in large-scale structures, and consequently infer information about cosmic magnetism (e.g., Beck R. et al. 2013, Gaensler et al. 2004). However, this is a non-trivial task that demands a statistical approach. The first statistical studies to prove the existence of Faraday rotation from large-scale extragalactic environments (galaxy clusters) were published by Lawler \& Dennison (1982) and Vallée et al. (1986). Later, an important statistical RM analysis on intracluster magnetic fields was performed by Clarke et al. (2001, 2004). Relevant contributions regarding intergalactic magnetic fields on even larger scales were done by Kolatt (1998), Stasyszyn et al. (2010), and Akahori et al. (2014).

A statistical theory for Bayesian inference on spatially distributed signals, Information Field Theory, was developed by Enßlin et al. (2009). Within the framework of this theory, Oppermann et al. $(2012,2014)$ reconstructed the Galactic Faraday rotation foreground and estimated the extragalactic contribution. In this chapter we take a step forward, with a new algorithm, able to statistically separate the different extragalactic contributions, by combining RMs with information about the large-scale structure of the cosmic web. This approach will make it possible to study magnetic fields on large scales with the RM grid data that will be obtained with the new generation of radio interferometers (see the chapter by Johnston-Hollitt et al. 2014 in these proceedings).

\section{General description}

The probability density distribution of the extragalactic Faraday rotation measure along a given 
line of sight $i$ can be described with a Gaussian of mean $\left\langle\phi_{\mathrm{e}, i}\right\rangle$ and variance $\left\langle\phi_{\mathrm{e}, i}^{2}\right\rangle$

$$
\begin{aligned}
\left\langle\phi_{\mathrm{e}, i}\right\rangle & =0 \\
\left\langle\phi_{\mathrm{e}, i}^{2}\right\rangle & =a_{0}^{2} \int_{0}^{x_{i}} \int_{0}^{x_{i}} \mathrm{~d} x \mathrm{~d} x^{\prime} \frac{n_{\mathrm{e}}(x) n_{\mathrm{e}}\left(x^{\prime}\right)\left\langle B_{x}(x) B_{x}\left(x^{\prime}\right)\right\rangle}{(1+z(x))^{2}\left(1+z\left(x^{\prime}\right)\right)^{2}}
\end{aligned}
$$

where $a_{0}$ is a constant, $x_{i}$ is the proper distance of the source, $z$ is the redshift, $n_{\mathrm{e}}$ is the electron density, and $B_{x}$ is the component of the magnetic field along the line of sight. We can define a length scale $\lambda_{x}$, so that the auto-correlation tensor of the magnetic field $\left\langle B_{x}(x) B_{x}\left(x^{\prime}\right)\right\rangle$ on average gives significant contributions for $\left|x-x^{\prime}\right|<\lambda_{x}$, but not for $\left|x-x^{\prime}\right| \gg \lambda_{x}$. The strength of the magnetic field, its correlation length, and the electron density have a different value in each environment $j$, i.e., in voids, sheets, filaments, galaxy clusters, and the in radio source itself. If we define

$$
\chi_{x}=a_{0}^{2} n_{\mathrm{e}}(x)^{2}\left\langle B_{x}(x)^{2}\right\rangle \lambda_{x}
$$

which we assume to depend only on the environment, so that $\chi_{x}=\chi_{j(x)}$, and introduce $l_{i j}$ as the length of the line of sight $i$ through each environment $j$, the overall variance in Faraday rotation can be written as

$$
\left\langle\phi_{\mathrm{e}, i}^{2}\right\rangle \approx a_{0}^{2} \int_{0}^{x_{i}} \frac{n_{\mathrm{e}}(x)^{2}\left\langle B_{x}(x)^{2}\right\rangle \lambda_{x}}{(1+z(x))^{4}} \mathrm{~d} x \approx \chi_{0}+\sum_{j} l_{i j} \chi_{j} .
$$

The intrinsic contribution of the emitting source $\chi_{0}$ is assumed to be on average the same for all sources. Since the intrinsic size of the emitting radio source is unknown, $\chi_{0}$ is defined according to Eq. (2.2) but with the length $l_{i 0}$ factored in. Here, the auto-correlation function of the magnetic field has been approximated with a top-hat function of width $\lambda_{x}$. We assume that, within a correlation length $\lambda_{x}$, the redshift can be approximated to be constant. Moreover, we note that $\chi_{j}$ has been assumed to be independent of the redshift, meaning that the contribution to the Faraday rotation from a given environment does not change with redshift. The redshift dependence is totally absorbed by the term $l_{i j}$. Residual small-scale, uncorrelated Galactic contributions could be present in the estimate of the extragalactic Faraday rotation $\phi_{\mathrm{e}, i}$. To properly take them into account, the inclusion of a latitude dependent contribution in Eq. (2.3) could be necessary.

To constrain the contribution from the different environments $\chi_{j}$, an estimate of the extragalactic Faraday rotation $\phi_{\mathrm{e}, i}$ for a collection of lines of sight, as well as a description of the cosmic web large-scale structure, summarized by $l_{i j}$, is required. With a Bayesian approach, Jasche et al. (2010) reconstructed the cosmic density field on the basis of optical data from the SDSS Data Release 7. Following the scheme proposed by Hahn et al. (2007), they give a three-dimensional classification of the density field posterior in terms of voids, sheets, filaments, and clusters of galaxies. For a source with known redshift, this reconstruction allows one to estimate the path covered by the radio signal through each environment, i.e., the elements $l_{i j}$ in Eq. (2.3). Radio sources can sit in different structures, e.g. galaxy clusters or filaments. This effect can be taken into account statistically since it is possible to locate the radio source in the reconstruction of the large scale structure via redshift identification. The fractions of the line of sight that the different radio sources spend in the different environments varies (this is also the case for the same radio source when different reconstruction of the large scale structure are considered). The combination of this information with 
the Faraday depth estimation for a collection of sources enables us therefore to statistically discriminate between the average contributions from the source itself, from galaxy clusters, filaments, voids, and sheets.

\section{Bayesian approach}

To infer information about the contribution from the different extragalactic environments, $\chi$, to the extragalactic Faraday rotation on the basis of data $d^{1}$ obtained from a RM grid, we propose a Bayesian approach. Bayes theorem,

$$
P(\chi \mid d)=\frac{P(d \mid \chi) P(\chi)}{P(d)}
$$

expresses our a posteriori knowledge $P(\chi \mid d)$ about the signal $\chi$ after the measurement process took place. The initial knowledge on the signal before the data have been acquired is described by the prior $P(\chi)$ and is modified by the data $d$ through the likelihood $P(d \mid \chi)$. The evidence $P(d)$ is a normalization factor, obtained by marginalizing the joint probability $P(d, \chi)=P(d \mid \chi) P(\chi)$ over all the possible configurations of the signal $\chi$.

In order to get a data-driven solution and to keep our assumptions as general as possible, we do not put constraints on the magnetic field strength and assume all values of magnetic field magnitudes to have equal probability. Such a priori knowledge can be well described by a flat prior on a logarithmic scale. Mathematically, it is expressed as an Inverse-Gamma distribution

$$
P(\chi) \propto \prod_{j} \chi_{j}^{-\alpha_{j}} e^{-\frac{q_{j}}{\chi_{j}}}
$$

where $\alpha_{j}=1$ and $q_{j}=0$ (Jeffreys' Prior).

The Faraday depths $d_{i}$ coming from radio observations contain the Galactic and extragalactic contribution, $\phi_{\mathrm{g}, i}$ and $\phi_{\mathrm{e}, i}$, and the noise $n_{i}$ of the measurement process

$$
d_{i}=\phi_{\mathrm{g}, \mathrm{i}}+\phi_{\mathrm{e}, \mathrm{i}}+n_{i}
$$

Oppermann et al. $(2012,2014)$ developed an algorithm to reconstruct the Galactic Faraday rotation foreground and estimate the extragalactic contribution, given a catalog of Faraday RMs for different lines of sight. Their results are available as a collection of $N$ random samples extracted from the posterior distribution

$$
P\left(\phi_{\mathrm{e}} \mid d, \mathrm{Opp}\right)=\frac{P\left(d \mid \phi_{\mathrm{e}}\right) P\left(\phi_{\mathrm{e}} \mid \mathrm{Opp}\right)}{P(d \mid \mathrm{Opp})},
$$

which intrinsically depends on the assumption enclosed in their prior, as indicated by "Opp". In order to disentangle the different contributions that make up the overall observed extragalactic Faraday depth, this prior must be replaced with a new one.

\footnotetext{
${ }^{1}$ Here, $d$ and $\chi$ are respectively vectors with elements $d_{i}$ and $\chi_{j}$ with $i=1, \ldots, N_{\text {los }}$ and $j=0, \ldots, N_{\mathrm{env}}$, where $N_{\text {los }}$ and $N_{\mathrm{env}}$ are the total number of lines of sight and the total number of environments in between the source and the observer.
} 
If in Eq. (3.1), we expand our likelihood

$$
P(d \mid \chi)=\int \mathscr{D} \phi_{\mathrm{e}} P\left(d \mid \phi_{\mathrm{e}}\right) P\left(\phi_{\mathrm{e}} \mid \chi\right)
$$

and insert the likelihood $P\left(d \mid \phi_{\mathrm{e}}\right)$ from Oppermann et al. (2014), see Eq. (3.4), we obtain

$$
P(\chi \mid d)=\int \mathscr{D} \phi_{\mathrm{e}} \frac{P\left(\phi_{\mathrm{e}} \mid d, \mathrm{Opp}\right) P(d \mid \mathrm{Opp})}{P\left(\phi_{\mathrm{e}} \mid \mathrm{Opp}\right)} \frac{P\left(\phi_{\mathrm{e}} \mid \chi\right) P(\chi)}{P(d)},
$$

where $\int \mathscr{D} \phi_{\mathrm{e}}$ denotes the phase space integral over all the possible field configurations of $\phi_{\mathrm{e}}$. As the evidence is simply a normalization factor, this posterior no longer depends on the assumptions of Oppermann et al. (2014), which cancel out.

Under the assumption that the lines of sight are sufficiently separated, that is, the crosscorrelation function of their magnetic field is zero, for each line of sight we can consider independent Gaussian distributions for the extragalactic Faraday depth given the cosmic web's large-scale structure

$$
P\left(\phi_{\mathrm{e}} \mid \chi\right)=\int \mathscr{D} l P(l \mid \mathrm{Jasche}) \prod_{i} \mathscr{G}\left(\phi_{\mathrm{e}, \mathrm{i}}, \sum_{\mathrm{j}} l_{i j} \chi_{j}\right),
$$

where $P(l \mid$ Jasche $)$ is the posterior of the cosmic web structure from Jasche et al. (2010), available in the form of $N^{\prime}$ samples. The notation $\mathscr{G}(x, X)$ indicates a one-dimensional Gaussian distribution for a variable $x$ with zero mean and variance $X$. Moreover, since the prior $P\left(\phi_{\mathrm{e}} \mid \mathrm{Opp}\right)$ from Oppermann et al. (2014) is

$$
P\left(\phi_{\mathrm{e}} \mid \mathrm{Opp}\right)=\prod_{i} \mathscr{G}\left(\phi_{\mathrm{e}, i}, \sigma_{\mathrm{e}}^{2}\right)
$$

with a standard deviation $\sigma_{\mathrm{e}} \sim 6.4 \mathrm{rad} / \mathrm{m}^{2}$, it follows that

$$
P\left(\chi, \phi_{\mathrm{e}}, l \mid d\right) \approx \frac{\prod_{i} \mathscr{G}\left(\phi_{\mathrm{e}, i}, \sum_{j} l_{i j} \chi_{j}\right)}{\prod_{i} \mathscr{G}\left(\phi_{\mathrm{e}, i}, \sigma_{\mathrm{e}}^{2}\right)} \prod_{j} \chi_{j}^{-1} P\left(\phi_{\mathrm{e}} \mid d, \text { Opp }\right) P(l \mid \text { Jasche }) .
$$

This probability density function can be marginalized over $l$ and $\phi_{\mathrm{e}}$ by making use of the samplerepresentation available for the posterior of the large scale structure $P(l \mid$ Jasche $)$ and of the extragalactic Faraday rotation $P\left(\phi_{\mathrm{e}} \mid d, \mathrm{Opp}\right)$. To evaluate the posterior distribution for $\chi$, we plan to use a Monte Carlo Markov Chain approach.

In this approximation the lines of sight are considered independent. This means that, if the assumption does not hold, a correlated component of the magnetic field could be missed. A proper modeling that takes into account possible cross-correlations between lines of sight is left for future works.

\section{Current developments and applications with SKA}

An application to archival Very Large Array (VLA) data is in progress. By reanalyzing $1.4 \mathrm{GHz}$ data from the NRAO VLA Sky Survey (NVSS), Taylor et al. (2009) derived a catalog of RM values for 37543 linearly polarized radio sources, north of declination $-40^{\circ}$ - which corresponds to a 
number density of approximately one source per square degree. Oppermann et al. (2014) evaluated the posterior distribution of the extragalactic Faraday rotation for all the lines of sight in this catalog.

By using optical data from on-line databases (NED and SIMBAD) as well as from surveys (SDSS, 6dFGS, 2dFGRS, 2QZ, 6QZ), Hammond et al. (2012) published a catalog of spectroscopic redshifts for 4003 linearly polarized sources from the NVSS. The sources in this sample have redshifts in the range $0.0007<z<5.3$. Only about 30 sources have $z<0.02$, and the mean redshift of the sample is 0.89 . The mean angular distance of the sources being equal to $\sim 1$ degree, this implies a mean distance among sources larger than $1 \mathrm{Mpc}$ for $99 \%$ of the sample, and on average larger than $\approx 30 \mathrm{Mpc}$. If $\lambda_{x}>>30 \mathrm{Mpc}$, the assumption of no cross-correlation is not valid anymore. As noted in the previous section, this would translate in an underestimate of the magnetic field for structures with such a length scale. By combining the reconstruction of the large-scale structure from Jasche et al. (2010) with this catalog of redshifts, it is possible to evaluate the path covered by the radio signal through each environment for each line of sight.

The catalog of Faraday rotation values in Taylor et al. (2009) was derived through a linear fit of the observed polarization angle versus $\lambda^{2}$ at two closely separated radio frequencies (1365 and $1435 \mathrm{MHz}$ ). When few data points are used, such $\lambda^{2}$-fits can suffer from an $n \pi$-ambiguity. Moreover, this approach is suitable only if no Faraday rotating plasma is mixed with the radio-emitting plasma. A better analysis of polarization properties of radio sources can be done with the RM synthesis (Brentjens \& de Bruyn 2005) and the Faraday synthesis (Bell \& Enßlin 2012) techniques if more frequencies are available. A continuous and broad frequency coverage is desirable, both to reduce the risk of $n \pi$-ambiguities and to reach a sufficiently high resolution in Faraday depth to distinguish nearby Faraday components (e.g., Farnsworth et al. 2011, Kumazaki et al. 2014).

In this respect, the Square Kilometer Array as well as its precursors will offer a great improvement. Broadband spectro-polarimetric surveys of the sky in combination with deep observations of specific targets will provide densely spaced RM grids. A RM catalog one hundred times denser than those currently existing (Taylor et al. 2009) will be obtained with POSSUM, the polarization survey planned with the SKA precursor ASKAP. POSSUM will observe the sky between 1130 and $1430 \mathrm{MHz}$ with a sensitivity in $\mathrm{U}$ and $\mathrm{Q}$ of $\sim 10 \mu \mathrm{Jy} / \mathrm{beam}$ and a resolution of $10^{\prime \prime}$, giving an expected density of 70 polarized sources per square degree (Hales et al. 2014a). The uncertainty on the RM is lower than $\approx 6 \mathrm{rad} / \mathrm{m}^{2}$ for a polarized signal with a $S / N>5$ (Stepanov et al. 2008). The ultra-deep polarization survey CHILES Con Pol with the Jansky Very Large Array (Hales et al. 2014b) will reach a sensitivity of $400 \mathrm{nJy}$ with sky area of a fraction of a square degree, providing a first look at the magnetic field science that can be performed with the SKA. The sky survey in polarization SKA1-SUR planned for SKA1 will go much further. Designed to cover the frequency range $650-1670 \mathrm{MHz}$, it will produce Stokes U and Q images over 30000 square degrees with a spatial resolution of $2^{\prime \prime}$. Within two years, a sensitivity in polarization of $\sim 1 \mu \mathrm{Jy} /$ beam will be reached, with an uncertainty in RM lower than $\approx 1 \mathrm{rad} / \mathrm{m}^{2}(S / N>5)$. The high RM accuracy will make our approach viable for detecting magnetic fields with strengths $\sim \mathrm{nG}$ already during the early science phase of SKA1, when sensitivity will be at $50 \%$ of its full level. For weaker magnetic fields the full deployment of all the SKA1 baseline will be necessary. The resulting sky grid of Faraday rotation values will be 300-1000 times denser than the largest catalog available at the moment. The better resolution of $2^{\prime \prime}$, compared to the $45^{\prime \prime}$ of Taylor et al. (2009), will make it possible to identify optical counterparts uniquely and hence the source redshift. For fields of particular interest, e.g., regions 
where galaxy filaments have been observed, deeper observations could be necessary to assess the presence of magnetic fields and their properties. In this case, the observations from the survey can be complemented by targeted SKA1-MID pointings over a continuous frequency band from 350 to $3050 \mathrm{MHz}$. This would imply a polarization sensitivity $\lesssim 1 \mu \mathrm{Jy} / \mathrm{beam}$ after one hour of integration time and an uncertainty in RM lower than $\approx 0.2 \mathrm{rad} / \mathrm{m}^{2}(S / N>5)$. These targeted observations will be crucial, both to increase the resolution in Faraday depth and to detect a larger number of sources, allowing to distinguish nearby components in Faraday spectra and improve the statistics.

The even higher spatial and frequency resolution and the deeper sensitivity of SKA2 will make it possible to obtain RMs for an unprecedented number of background and intervening sources. It is therefore essential to develop and apply statistical approaches with the aim of interpreting the data correctly.

\section{Conclusions}

The origin and evolution of cosmological magnetic fields are still open questions. Magnetic fields are currently being studied in galaxy clusters but a firm detection is lacking in larger scale structures, such as galaxy filaments. The structure and the strength of magnetic fields in galaxy clusters are both deeply affected by the cluster formation processes (e.g., Dolag et al. 2005, Donnert et al. 2009, Xu et al. 2010), almost independently of the mechanism of generation of the seed magnetic field. This makes it difficult to discriminate between the different formation scenarios from galaxy cluster observations alone. The SKA's large bandwidth, high sensitivity and frequency resolution will permit a more reliable recovery of the Faraday depth for a large number of sources, and its high spatial resolution will allow a proper identification of the optical counterparts necessary for redshift assignment. Modern techniques based on the largest photometric all-sky surveys (2MASS, WISE and SuperCOSMOS) already provide catalogs of redshifts for millions of sources up to redshifts of 0.2 to 0.3 (e.g. Bilicki et al. 2014), and the next generation of redshift surveys will push those limits. We will investigate to what depths and accuracy redshifts are needed in order to apply the proposed method. The ultimate goal is to search for magnetic fields in largescale structures like filaments, where magnetic field amplification is not yet saturated and therefore the field strength still depends on the seed field strength. This information will help to place firm constraints on the origin and evolution of cosmological magnetic fields.

\section{Acknowledgments}

We thank the anonymous referee for useful comments and suggestions. The implementation of the code makes use of the NIFTY package by Selig et al. (2013) and of the cosmology calculator by Ned Wright (www.astro.ucla.edu/ wright). This research was supported by the DFG Forschengruppe 1254 "Magnetisation of Interstellar and Intergalactic Media: The Prospects of Low-Frequency Radio Observations".

\section{REFERENCES}

Akahori, T., Gaensler, B. M., \& Ryu, D. 2014, ApJ, 790, 123 
Beck, A. M., Hanasz, M., Lesch, H., Remus, R.-S., \& Stasyszyn, F. A. 2013, MNRAS, 429, L60

Beck, R., Anderson, J., Heald, G., et al. 2013 334, 548

Bell, M. R., \& Enßlin, T. A. 2012, A\&A, 540, A80

Bilicki, M., Peacock, J. A., Jarrett, T. H., Cluver, M. E., \& Steward, L. 2014, arXiv:1408.0799

Bonafede, A., Feretti, L., Giovannini, G., et al. 2009, A\&A, 503, 707

Bonafede, A., Feretti, L., Murgia, M., et al. 2010, A\&A, 513, A30

Brentjens, M. A., \& de Bruyn, A. G. 2005, A\&A, 441, 1217

Carilli, C. L., \& Taylor, G. B. 2002, ARA\&A, 40, 319

Clarke, T. E., Kronberg, P. P., Böhringer, H. 2001, ApJL, 547, L111

Clarke, T. E. 2004, Journal of Korean Astronomical Society, 37, 337

Dolag, K., Grasso, D., Springel, V., \& Tkachev, I. 2005, JCAP, 1, 9

Dolag, K., Bykov, A. M., \& Diaferio, A. 2008, SSRv, 134, 311

Donnert, J., Dolag, K., Lesch, H., Müller, E. 2009, MNRAS, 392, 1008

Enßlin, T. A., \& Vogt, C. 2003, A\&A, 401, 835

Enßlin, T. A., Frommert, M., \& Kitaura, F. S. 2009, PhRvD, 80, 105005

Farnsworth, D., Rudnick, L., \& Brown, S. 2011, AJ, 141, 191

Gaensler, B. M., Beck, R., \& Feretti, L. 2004, NewAR, 48, 1003

Giovannini, G. et al. 2015, "Cluster magnetic fields through the study of polarized radio halos in the SKA era", in proc. Advancing Astrophysics with the Square Kilometre Array, PoS(AASKA14) 105

Govoni, F., \& Feretti, L. 2004, International Journal of Modern Physics D, 13, 1549

Govoni, F., Murgia, M., Feretti, L., et al. 2006, A\&A, 460, 425

Guidetti, D., Murgia, M., Govoni, F., et al. 2008, A\&A, 483, 699

Hahn, O., Porciani, C., Carollo, C. M., \& Dekel, A. 2007, MNRAS, 375, 489

Hales, C. A., Norris, R. P., Gaensler, B. M., \& Middelberg, E. 2014a, MNRAS, 440, 3113

Hales, C. A., \& Chiles Con Pol Collaboration 2014b, Exascale Radio Astronomy, 50301

Hammond, A. M., Robishaw, T., \& Gaensler, B. M. 2012, arXiv:1209.1438

Jasche, J., Kitaura, F. S., Li, C., \& Enßlin, T. A. 2010, MNRAS, 409, 355

Johnston-Hollitt, M. et al. 2015, "Using SKA Rotation Measures to Reveal the Mysteries of the Magnetised Universe", in proc. Advancing Astrophysics with the Square Kilometre Array, PoS(AASKA14)092

Kolatt, T. 1998, ApJ, 495, 564

Kumazaki, K., Akahori, T., Ideguchi, S., Kurayama, T., \& Takahashi, K. 2014, PASJ, 66, 61

Lawler, J. M., \& Dennison, B. 1982, ApJ, 252, 81

Murgia, M., Govoni, F., Feretti, L., et al. 2004, A\&A, 424, 429

Oppermann, N., Junklewitz, H., Robbers, G., et al. 2012, A\&A, 542, A93

Oppermann, N., Junklewitz, H., Greiner, M., et al. 2014, arXiv:1404.3701

Selig, M., Bell, M. R., Junklewitz, H., et al. 2013, A\&A, 554, A26

Stasyszyn, F., Nuza, S. E., Dolag, K., Beck, R., \& Donnert, J. 2010, MNRAS, 408, 684

Stepanov, R., Arshakian, T. G., Beck, R., Frick, P., \& Krause, M. 2008, A\&A, 480, 45

Taylor, A. R., Stil, J. M., \& Sunstrum, C. 2009, ApJ, 702, 1230

Vacca, V., Murgia, M., Govoni, F., et al. 2010, A\&A, 514, A71

Vacca, V., Murgia, M., Govoni, F., et al. 2012, A\&A, 540, A38 
Vallée, J. P., MacLeod, M. J., \& Broten, N. W. 1986, A\&A, 156, 386

Vogt, C., \& Enßlin, T. A. 2003, A\&A, 412, 373

Vogt, C., \& Enßlin, T. A. 2005, A\&A, 434, 67

Xu, H., Li, H., Collins, D. C., Li, S., \& Norman, M. L. 2010, ApJ, 725, 2152 